\title{
Cosmic rays: extragalactic and Galactic
}

\section{Istomin Ya.N.}

Moscow Institute Physics and Technology,

Institutskii per. 9, Dolgoprudnyi, Moscow region, 141700, Russia

istomin.ian@mipt.ru, istomin@lpi.ru

From the analysis of the flux of high energy particles, $E>3 \cdot 10^{18} \mathrm{eV}$, it is shown that the distribution of the power density of extragalactic rays over energy is of the power law, $\bar{q}(E) \propto E^{-2.7}$, with the same index of 2.7 that has the distribution of Galactic cosmic rays before so called 'knee', $E<3 \cdot 10^{15} \mathrm{eV}$. However, the average power of extragalactic sources, which is of $\mathscr{E} \simeq 10^{43} \mathrm{erg} \mathrm{s}^{-1}$, at least two orders exceeds the power emitted by the Galaxy in cosmic rays, assuming that the density of galaxies is estimated as $N_{g} \simeq 1 \mathrm{Mpc}^{-3}$. Considering that such power can be provided by relativistic jets from active galactic nuclei with the power $\mathscr{E} \simeq 10^{45}-10^{46} \mathrm{erg} \mathrm{s}^{-1}$, we estimate the density of extragalactic sources of cosmic rays as $N_{g} \simeq 10^{-2}-10^{-3} \mathrm{Mpc}^{-3}$. Assuming the same nature of Galactic and extragalactic rays, we conclude that the Galactic rays were produced by a relativistic jet emitted from the Galactic center during the period of its activity in the past. The remnants of a bipolar jet are now observed in the form of bubbles of relativistic gas above and below the Galactic plane. The break, observed in the spectrum of Galactic rays ('knee'), is explained by fast escape of energetic particle, $E>3 \cdot 10^{15} \mathrm{eV}$, from the Galaxy because of the dependence of the coefficient of diffusion of cosmic rays on energy, $D \propto E^{0.7}$. The obtained index of the density distribution of particles over energy, $N(E) \propto E^{-2.7-0.7 / 2}=E^{-3.05}$, for $E>3 \cdot 10^{15} \mathrm{eV}$ agrees well with the observed one, $N(E) \propto E^{-3.1}$. Estimated time of termination of the jet in the Galaxy is $4.2 \cdot 10^{4}$ years ago.

The 34th International Cosmic Ray Conference,

30 July- 6 August, 2015

The Hague, The Netherlands 


\section{Introduction}

The main hypothesis of the origin of cosmic rays in the Galaxy is the acceleration of charged particles to high energies on the fronts of shock waves formed by supernova explosions. The observed spectrum over energy differs from the spectrum given by sources, remaining a power law, $N(E) \propto E^{-\beta}$. But the value of $\beta$ is not constant, it changes from $\beta_{1}=2.7$ for $E<3 \cdot 10^{15} \mathrm{eV}$ to $\beta_{2}=3.1$ for $E>3 \cdot 10^{15} \mathrm{eV}$. The important circumstance is the fact that the spectrum at high energies becomes softer, not harder. This suggests that the source of cosmic rays at energies $E<3$. $10^{15} \mathrm{eV}$ and at energies $E>3 \cdot 10^{15} \mathrm{eV}$ is single. It is possible the superposition of two independent sources, if it would be vice versa, $\beta_{2}<\beta_{1}$. But it is unlikely that the spectrum produced by one source at $E<3 \cdot 10^{15} \mathrm{eV}$ would cut off at higher energies, whereas another independent source at energies $E>3 \cdot 10^{15} \mathrm{eV}$ would cut off at lower energies, and they were joined at the same energy $E \simeq 3 \cdot 10^{15} \mathrm{eV}$.

Here we do not discuss the paradigm of acceleration of Galactic cosmic rays by shock waves from supernova explosions. Last review made by Blasi (2013) gives a good representation of this paradigm in many aspects. Observational data certainly show the acceleration of particles on fronts of supernova shock waves. Is that enough to consider a hypothesis of the origin of Galactic cosmic rays by shock wave acceleration is proven? Here you can quote from the Blasi's review: "Despite all this circumstantial evidence, no proof found yet that SNRs can accelerate CRs up to the knee energy."

If the source is single, it would produce cosmic rays also in other galaxies with characteristics similar to those observed in the Galaxy. The aim of our work is to analyze the properties of cosmic rays of superhigh energies $E>3 \cdot 10^{18} \mathrm{eV}$ observed on the Earth, coming outside, from other galaxies, and compare them with the properties of Galactic cosmic rays.

\section{Energy distribution of extragalactic particles observed in the Galaxy}

The solution of the kinetic equation, describing the cosmic rays propagation in an intergalactic space, is

$$
N(\varepsilon)=\frac{\int_{\varepsilon}^{\varepsilon_{0}} \bar{q}\left(\varepsilon^{\prime}\right) d \varepsilon^{\prime}}{|d \varepsilon / d t|}, \varepsilon=\frac{E}{4 \cdot 10^{20} e V} .
$$

Here $N(\varepsilon)$ is the distribution function of extragalactic cosmic rays observed in the Galaxy, $\bar{q}(\varepsilon)$ is the power denisity of cosmic rays sources, $d \varepsilon / d t$ is the energy losses of particles. Equation (2.1) has a simple physical meaning: the particle flux in the energy space, $N|d \varepsilon / d t|$, is equal to the total flux produced by sources $Q_{i}$. Moreover, since we have many sources, their fluxes are summing, ranging from close sources, providing a full range of energies from $\varepsilon=\varepsilon_{\min }$ up to the maximum one $\varepsilon=\varepsilon_{0}$, to distant sources from which we observe only large initial energy $\varepsilon \simeq \varepsilon_{0}$. Suppose that the distribution $\bar{q}(\varepsilon)$ is the power law function, $\bar{q}(\varepsilon)=q_{0} \varepsilon^{-\beta}$. Then the distribution function $N(\varepsilon)$ is equal to

$$
\begin{aligned}
& N(\varepsilon)=\frac{q_{0}}{\beta-1} \frac{\varepsilon^{-\beta+1}-\varepsilon_{0}^{-\beta+1}}{F(\varepsilon)} \\
& F(\varepsilon)=(1+\varepsilon) \exp (-1 / \varepsilon)+a \varepsilon\left(\varepsilon_{e}^{-2}+b \varepsilon_{e}^{0.6}\right)^{-1} \exp \left(-1 / \varepsilon_{e}\right)+\alpha \varepsilon, \varepsilon_{e}=\frac{E}{2.1 \cdot 10^{18} e V} .
\end{aligned}
$$




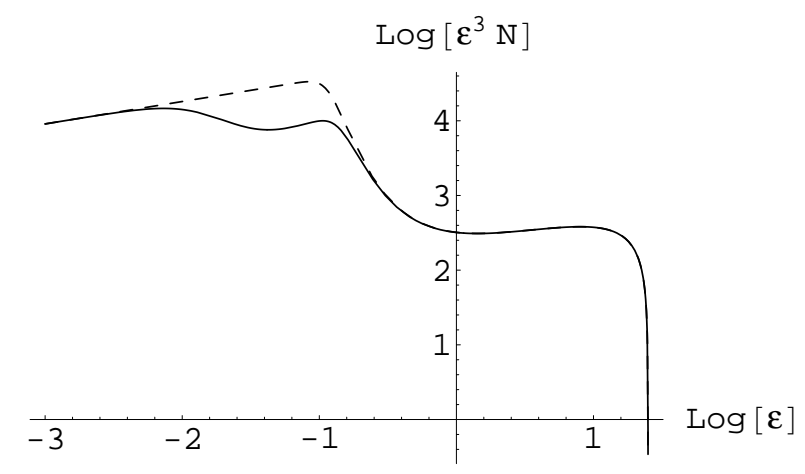

Figure 1: The function $\varepsilon^{3} N(\varepsilon), N(\varepsilon)$ is given by the formula (2.2). Here we choose the value of maximum energy $E_{0}=10^{22} \mathrm{eV}, \varepsilon_{0}=25$, and the index $\beta=2.7$. The dashed line represents this function when the electron-positron pairs production is absent.

The graph of the function $\varepsilon^{3} N(\varepsilon)$ for $\varepsilon_{0}=25, E_{0}=10^{22} \mathrm{eV}$, and $\beta=2.7$ is shown on Fig. 1 . One can see that at energies $E<10^{18} \mathrm{eV}$ and $E>4 \cdot 10^{20} \mathrm{eV}$ the distribution $N(\varepsilon)$ is the power law, $N(\varepsilon) \propto \varepsilon^{-\beta}$. The same slope is observed at the intermediate energies, in the region of the maximum relative losses of energy, $(d E / d t) / E$, on the birth of electron-positron pairs, $E \simeq$ $10^{19} \mathrm{eV}$. In the region $0.2<\varepsilon<1, N$ grows exponentially with decreasing of energy, $N(\varepsilon)=$ $q_{0} \varepsilon^{-\beta+1} \exp (1 / \varepsilon) /(\beta-1)$. According to observations summarized by Berezinsky (2013), the flux of particles $I(\varepsilon)=c N(\varepsilon) / 4 \pi$ in the energy range $6 \cdot 10^{18} \mathrm{eV}<E<4 \cdot 10^{19} \mathrm{eV}, 1.5 \cdot 10^{-2}<\varepsilon<0.1$, is indeed the power law function of the energy with the index $\beta=2.7$. Berezinsky (2014) showed that the actual energies are required to move a little upwards. This corresponds to the range $6 \cdot 10^{18} \mathrm{eV}<E<4 \cdot 10^{19} \mathrm{eV}$. On the Fig. 1 this energy is below the maximum at $E=4 \cdot 10^{19} \mathrm{eV}$. When shifting energies the slope does not change and remains 0.3 .

The function $\varepsilon^{3} N(\varepsilon)$ has a maximum at $\varepsilon=\varepsilon_{1}=0.1, E_{1}=4 \cdot 10^{19} \mathrm{eV}$ (see Fig. 1). At energies $\varepsilon>\varepsilon_{1}$ the function $\varepsilon^{3} N(\varepsilon)$ exponentially decreases not to zero, but to the minimum value at $\varepsilon_{2}$, $\varepsilon_{2} \simeq 1, E_{2} \simeq 4 \cdot 10^{20} \mathrm{eV}$. After that the distribution $N(\varepsilon)$ continues to fall down by a power law manner with the same index $\beta, N(\varepsilon) \propto \varepsilon^{-\beta}$. The fall down of the function $\varepsilon^{3} N(\varepsilon)$ at energy $\varepsilon_{1}$ to its value at $\varepsilon_{2}$ is approximately one and half orders. The strong growth of the density of particles for $\varepsilon<\varepsilon_{2}$ is explained by the sharp decreasing of the rate of energy losses of particles when braking by relict photons becomes small. Here particles are accumulated. The distribution of $N(E)$ for energies $E>4 \cdot 10^{20} \mathrm{eV}$ does not go exponentially to small values that can seem as the result of the GZK effect. Here we observe that the distribution of $N(E)$ repeats the distribution of extragalactic sources $\bar{q}(E)$, unless of the maximum energy $E_{0}$ is not close to the energy $4 \cdot 10^{20} \mathrm{eV}$. However, at energies $E>4 \cdot 10^{20} \mathrm{eV}$ the observational data have great uncertainty, which does not allow to make a conclusion about the growth of the function $E^{3} N(E)$ at high energies $E>4 \cdot 10^{20} \mathrm{eV}$ under the condition $E_{0}>4 \cdot 10^{20} \mathrm{eV}$. The energy range $E<3 \cdot 10^{18} \mathrm{eV}$ (see Fig. 1), where the distribution $N(E)$ also reproduces the distribution of extragalactic sources $\bar{q}(E)$, is hidden by Galactic cosmic rays.

Let us estimate now the average power density of sources $\bar{q}$. Observations give the value of the extragalactic particle flux, where there is maximum value of product $E^{3} I(E)$ at the energy 
$E_{1}=4 \cdot 10^{19} \mathrm{eV}$

$$
I\left(E_{1}\right)=4.7 \cdot 10^{-39} \mathrm{~cm}^{-2} \mathrm{~s}^{-1} \mathrm{sr}^{-1} \mathrm{eV}^{-1} .
$$

Considering that, as well as in our Galaxy, the power law spectrum with index $\beta=2.7$ continues up to small energies $E_{\min } \simeq 5 \mathrm{GeV}$, from the equation (2.1) we get

$$
\bar{q}(E)=8.4 \cdot 10^{8}\left(\frac{E}{4 \cdot 10^{19} e V}\right)^{-2.7} e V^{-1} s^{-1} M p c^{-3} .
$$

The total average power density of extragalactic sources $\mathscr{E}=\int_{E_{\min }}^{E_{0}} \bar{q}(E) E d E$ is equal to

$$
\mathscr{E}=1.8 \cdot 10^{43}\left(\frac{E_{\min }}{5 G e V}\right)^{-0.7} \operatorname{erg~s}^{-1} \mathrm{Mpc}^{-3}
$$

This value divided by the density of galaxies, $N_{g} \simeq 1 \mathrm{Mpc}^{-3}$, at least two orders of magnitude exceeds the power of the Galaxy in cosmic rays, $10^{41} \mathrm{erg} \mathrm{s}^{-1}$. If we assume that the sources of ultrahigh energy particles are active galactic nuclei with relativistic jets generated inside, the power of which is $\left(10^{45}-10^{46}\right) \operatorname{ergs}^{-1}$ (Mao-Li, et al., 2008), then their density in the Universe is of $N_{g} \simeq\left(10^{-2}-10^{-3}\right) M p c^{-3}$. The same estimation of the density of extragalactic sources of cosmic rays follows also from the conditions of isotropy of arrival of particles in the range of energies $E \simeq 10^{19} \mathrm{eV}$ (Abbasi et al., 2004).

\section{Galactic cosmic rays}

The most surprising fact, following from the previous consideration, is that the spectrum of the power density of extragalactic cosmic rays (2.2) has the the same slope that have Galactic cosmic rays density before the 'knee', $E<3 \cdot 10^{15} \mathrm{eV}, \beta=2.7$. This indicates that the nature of the origin of cosmic rays in the Galaxy and in active galactic nuclei is the same. The formation of the spectrum with index $\beta=2.7$ in the Galaxy is explained by the fact that the index of the power law energy spectrum of source is of universal value of $\beta=2$. This is valid as for the acceleration of particles on fronts of strong shock waves, as for the acceleration at the base of the jets emitted near massive black holes in centers of galaxies (Istomin, 2014). Next, accelerated particles, spreading over a galaxy, are scattered by inhomogeneities of a magnetic field. Their motion becomes diffusive. Moreover, the coefficient of diffusion is larger for particles with larger energies, $D \propto E^{\delta}$. It should be noted that the index $\delta$ is the same for protons and for different nuclei. Only diffusion coefficients can slightly vary at the same energy $E$. But the diffusion is the same for particles of the same rigidity $\mathscr{R}$ (Blasi \& Amato, 2012). The observed spectrum of Galactic cosmic rays under the assumption about the universality of the source gives the value of $\delta$ of 0.7 . We are taking this value as following from the observations, regardless of other values obtained from theoretical and numerical calculations of the scattering of charged particles and of the paradigm of particle acceleration by shock waves from supernova explosions.

The density of particles is equal to the product of the power of a source $Q$ to the lifetime of particles $\tau, N=Q \tau$. The lifetime is the time of escape of particles from a galaxy, $\tau=R^{2} / D, R$ is the radius of a galaxy. Thus, $N(E) \propto E^{-2-0.7}$. There arises the question: why in our Galaxy the spectral index of cosmic rays at energies $E>3 \cdot 10^{15} \mathrm{eV}$ deviates from the value of $\beta=2.7$ ? 


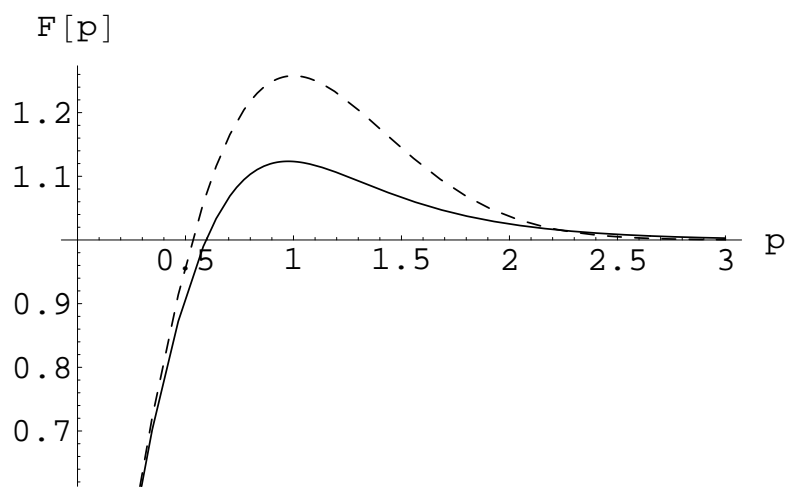

Figure 2: The function $F(p)$. The solid line corresponds to $r=R / 2$, the dashed line $-r=0$

Spectrum becomes softer, $\beta=3.1$. If the source of cosmic rays in a galaxy is strong shock waves from supernova explosions, it is not clear why the spectrum of high energy particles from active galaxies remains with index $\beta=2.7$ up to energies $\sim 10^{20} \mathrm{eV}$. More natural to assume that the source of cosmic rays is a jet emitted from active galactic nucleus and whose power significantly exceeds the power transformed to particles by supernova explosions. Istomin (2014) suggested that Galactic cosmic rays were produced by the jet, emitted from the center of the Galaxy. Giant bubbles of relativistic gas, observed above and below the Galactic plane, are remnants of this bipolar jet existed previously. From the size of the bubbles it follows that the jet switched on $2.4 \cdot 10^{7}$ years ago, and it worked at least $10^{7}$ years. Thus, before the jet switched off the Galaxy and its halo were uniformly filled by cosmic rays with the spectral index $\beta=2.7$ (Istomin, 2014). After the source turned off particles continue to flow out from the Galaxy, and their density begins to decrease with time. Consider how it is happen.

Suppose that at time $t=0$ cosmic rays with density $N_{0}(E)$ uniformly filled the spherical region (Galaxy and halo) of radius $R$. Then, assuming that the motion of particles is of diffusion character, we find the density of cosmic rays $N(r, E, t)$ at the point located at distance $r$ from the center of the Galaxy and at the time $t, N(r, E, t)=N_{0}(E) F(p, r)$, where the function $F(p, r)$ is the function of the parameter $p, p(E, t)=\left[R^{2} / 4 D_{g}(E) t\right]^{1 / 2}$. For small values of $p, p<<1$, the function $F$ is equal to $F=4 p / \pi^{1 / 2}$, for large values $p>>1, F=1$. The graph of the function $F(p)$ for two different values of $r=R / 2$ and $r=0$ are presented on the Fig. 2. It is seen that the transition of density of cosmic rays from the initial distribution $N_{0}(p>1)$ to the falling down with time, $N=$ $N_{0}\left(4 R^{2} / \pi D_{g} t\right)^{1 / 2}$, takes place at $p \simeq 1$. At this point there is a transition of the spectrum of cosmic rays from the original, $N \propto E^{-2.7}$, with energies $E<E_{k}$ to the distribution of $N \propto E^{-2.7-0.7 / 2}=$ $E^{-3.05}$ for $E>E_{k}$. The value of energy $E_{k}$ is defined by the equality $p=1, D_{g}\left(E_{k}\right)=R^{2} / 4 t$. In the center of the Galaxy $(r=0)$ the transition occurs exactly at the point $p=1$, for us $(r \simeq R / 2)$, this point is also very close to unity. When $p=1$, there is a local increase of density of cosmic rays, i.e. near the energy $E \simeq E_{k}$ the distribution $N(E)$ is really observed as 'knee'.

The position of the 'knee' depends on the time $t$, passed after turning off of the source. Knowing the position of the 'knee' at the present time $t=t_{0}$, we find

$$
t_{0}=\frac{R^{2}}{4 D_{g}\left(E_{k}\right)}=4.2 \cdot 10^{4}\left(\frac{R}{5 \cdot 10^{22} \mathrm{~cm}}\right)^{2}\left(\frac{D_{g}(1 \mathrm{GeV})}{2.2 \cdot 10^{28} \mathrm{~cm}^{2} \mathrm{~s}^{-1}}\right)^{-1}\left(\frac{E_{k}}{3 \cdot 10^{15} \mathrm{eV}}\right)^{-0.7} \text { year. }
$$


It should be noted that 'knee' is moving with time, $E_{k}=3 \cdot 10^{15}\left(t / t_{0}\right)^{-1.43} \mathrm{eV}$. The speed is now equal to $d E_{k} / d t=-1.43 E_{k} / t_{0}=-10^{11} \mathrm{eV} /$ year.

\section{Conclusions}

We showed that the distribution of power of sources of extragalactic cosmic rays in the energy range of $3 \cdot 10^{18} \mathrm{eV}<E<10^{21} \mathrm{eV}$ is a power law, $\bar{q}(E) \propto E^{-2.7}$. Thus, it is the same as in our Galaxy at the energy range below the 'knee', $E<3 \cdot 10^{15} \mathrm{eV}$. This indicates the common nature of the origin of cosmic rays in the Galaxy and in other galaxies. However, the power of extragalactic sources at least two orders of magnitude exceeds the capacity of the Galaxy (see formula (2.4)). The conclusion is that a 'normal' galaxy, to which belongs our Galaxy, is not the source of cosmic rays. The estimation of the density of extragalactic cosmic rays sources, $N_{g} \simeq 10^{-2}-10^{-3} \mathrm{Mpc}^{-3}$, indicates galaxies with active nuclei. The possible source of energetic charged particles is relativistic jets emitted from surroundings of massive black holes. Thus, acceleration by shock waves from supernova explosion does not explain the origin of cosmic rays up to energies $\simeq 10^{18} \mathrm{eV}$. Otherwise, all galaxies in more or less degree would are sources of cosmic rays, because in all of them there are explosions of supernova. Why, nevertheless, we observe cosmic rays in the Galaxy is explained that once in the past the Galaxy was also active. From the center of the Galaxy there emitted the relativistic bipolar jet, the remnants of which is observed now above and below the Galactic plane as bubbles of relativistic gas (Su, Slatyer \& Finkbeiner, 2010). The 'knee' formation reflects the escape of particles from the Galaxy. Knowing the position of the 'knee' now one can estimate the time when the source of cosmic rays in the Galaxy stopped, it occurred $4.2 \cdot 10^{4}$ years ago. The position of the 'knee' is not constant in time, it must move, $E_{k} \propto t^{-1.43}$. This motion one can notice if to have a sufficient accuracy of the measurement of the 'knee' position. During 50 years the change in the position of the 'knee' is $\Delta E_{k}=-5 \cdot 10^{12} \mathrm{eV}$.

\section{References}

[1] P. Blasi, The origin of galactic cosmic rays, The Astronomy and Astropart. Review 21 (2013) 70.

[2] V. Berezinsky, UHECR: Signatures and models, EPJ Web of Conference 53 (2013) 01003.

[3] V. Berezinsky, Extragalactic cosmic rays and their signatures, Astropart. Phys. 53 (2014) 120.

[4] M. Mao-Li, C. Xin-Wu, J. Dong-Rong, G. Min-Feng, A New Approach for Estimating Kinetic Luminosity of Jet in AGNs, Chin. J. Astron. Astrophys. 8 (2008) 39.

[5] R.U. Abbasi, et al., Study of Small-Scale Anisotropy of Ultra-High-Energy Cosmic Rays Observed in Stereo by the High Resolution Fly's Eye Detector, ApJ 610 (2004) L73.

[6] Ya.N. Istomin, On the origin of galactic cosmic rays, New Astronomy 27 (2014) 13.

[7] P. Blasi, E. Amato, emphDiffusive propagation of cosmic rays from supernova remnants in the Galaxy. II: anisotropy, JCaP, Issue 1, id. 011 (2012).

[8] M. Su, T.R. Slatyer and D.P. Finkbeiner, Giant Gamma-ray Bubbles from Fermi-LAT: Active Galactic Nucleus Activity or Bipolar Galactic Wind?, ApJ 724 (2010) 1044. 